\title{
Nach ESWL Tamsulosin geben?
}

Der Erfolg der extrakorporalen Stoßwellenlithotripsie bei symptomatischen Nierensteinen hängt von zwei Aspekten ab: zum einen ob es gelingt, den Stein ausreichend zu zerkleinern, und zum anderen ob die Fragmente problemlos den Harnleiter passieren. Eventuell kann man mit Medikamenten den zweiten Schritt entscheidend unterstützen.

$\mathrm{B}$ rasilianische Wissenschaftler haben in einer doppelblinden und placebokontrollierten Studie mit 111 Nierensteinpatienten untersucht, ob sich der Steinabgang nach einer extrakorporalen Stoßwellenlithotripsie (ESWL) durch die adjuvante Gabe von Tamsulsoin oder Nefedipin beschleunigen lässt. Die Urologen nahmen nur Patienten in die Studie auf, die älter als 18 Jahre waren, deren Steine röntgendicht und zwischen 5 und $20 \mathrm{~mm}$ groß waren und nicht im unteren Nierenpol lagen. Nach abgeschlossener ESWL erhielten 38 Patienten 0,4 mg Tamsulosin pro Tag, 35 Patienten $20 \mathrm{mg}$ Nifedipin und weitere 38 Placebo für maximal 30 Tage. Alle Patienten wurden wöchentlich kontrolliert und dann als erfolgreich therapiert eingestuft, wenn röntgenologisch keine Steine mehr nachzuweisen waren oder nur noch Fragmente von $4 \mathrm{~mm}$ oder kleiner zu finden waren.

Von den Patienten, die mit Tamsulosin behandelt worden waren, wurden $60,5 \%$ steinfrei, von den Nifedipin-Patienten 48,6\% und in der Placebo-Gruppe 36,8\%. Zwar war bei der Gesamtbetrachtung kein signifikanter Unterschied auszumachen $(\mathrm{p}=0,118)$, das änderte sich aber, wurden die Ergebnisse nochmals abhängig von der Steingröße (5-9 mm und 10-20 mm) bewertet. Patienten mit größeren Nierensteinen profitierten demnach durchaus von einer anschließenden medikamentösen Therapie, wobei beide Wirkstoffe ähnlich effektiv waren (Erfolgsraten: $61,9 \%$ unter Tamsulosin, $60 \%$ unter Nifedipin und $26,1 \%$ unter Placebo; $p=0,024)$. Allerdings litten unter Nifedipin mehr Patienten an Nebenwirkungen wie Kopfschmerzen und Benommenheit als unter Tamsulosin (28,5\% vs. $15,8 \%)$.

Auch wenn die Medikation den Therapieerfolg insgesamt verbesserte, schneller ging es damit nicht. Die Patienten mussten im Durchschnitt - egal ob mit oder ohne Medikation - etwas länger als zwei Wochen warten, bis die Steine endlich ausgespült wurden (Tage bis zum Steinabgang: 15,3 $\pm 2,1$ unter Tamsulosin, 15,9 $\pm 2,4$ unter Placebo, 16,7 $\pm 2,2$ unter Nifedipin). Auch die Schmerzintensität blieb von der Medikation unbeeinflusst. Der Schmerzmittelverbrauch war in allen drei Gruppen in etwa gleich hoch.

Fazit: Patienten, die nach einer ESWL mit dem Alpha-Rezeptorenblocker Tamsulosin oder dem Kalziumantagonisten Nifedipin behandelt werden, haben eine größere Chance, steinfrei zu werden, als solche, die keine medikamentöse Unterstützung erhalten. Das gilt jedoch nur für Steine, die ursprünglich zwischen $10 \mathrm{~mm}$ und $20 \mathrm{~mm}$ groß waren.

Dr. Dagmar Kraus

Vincentini FC et al. Adjuvant tamsulosin or nifedipine after extracorporeal shock wave lithotripsy for renal stones: a double blind, randomized, placebo-controlled trial. Urology 2011; 78: 1016-22

\section{Achtung:}

\section{Hier muss der Dummy durch eine Anzeige ersetzt werden !!}

